\title{
Sentiment Analysis about Smart Phones Using Twitter Corpus by Deep Learning Approach
}

\author{
R. Pavithra ${ }^{1}$ and A. R. Mohamed Shanavas ${ }^{2}$ \\ ${ }^{1}$ Research Scholar, ${ }^{2}$ Associate Professor, \\ ${ }^{1 \& 2}$ Department of Computer Science, Jamal Mohamed College, Trichy, Tamil Nadu, India \\ E-Mail: pavithra11746@gmail.com arms3375@gmail.com
}

\begin{abstract}
Micro blogging websites are nothing but social media website to which user makes quick and frequent posts. Twitter is one of the well-known micro blog sites which offer the space for person which can read and put up messages that are 148 characters in duration. Twitter messages also are referred to as Tweets. And will use these tweets as raw facts. Then use a way that automatically extracts tweets into advantageous, bad or neutral sentiments. By the usage of the sentiment evaluation the consumer can recognize the feedback about the product or services before make a purchase. The organization can use sentiment evaluation to know the opinion of clients about their products, so can examine customer pleasure and in line with that they could improve their product. Now-a-days social networking sites are at the growth, so massive amount of data is generated. Millions of human beings are sharing their views each day on micro blogging sites, since it includes short and simple expressions. In this thesis, able to discuss approximately a paradigm to extract the sentiment from a famous micro running a blog carrier, Twitter, wherein customers submit their opinions for the whole thing. And can use the deep mastering algorithm to categories the twitters which incorporates Convolutional Neural Networks. The experimental end result is presented to demonstrate the use and effectiveness of the proposed system.

Keywords: Social Networking, Micro Blogging, Deep Learning, Sentiment Analysis, Convolutional Neural Network
\end{abstract}

\section{INTRODUCTION}

Nowadays, the age of Internet has changed the way people express their views, opinions. It is now mainly done through blog posts, online forums, product review websites, social media. Millions of people are now using social network sites like Facebook, Twitter, Google Plus, etc. to express their emotions opinions and share views about their daily lives. Through the online communities, we get an interactive media where consumers inform and influence others through forums. Social media is generating a large volume of sentiment rich data in the form of tweets, status updates, blog posts, comments, reviews, etc. Moreover, social media provides an opportunity for businesses by giving a platform to connect with their customers for advertising. People mostly depend upon user generated content over online to a great extent for decision making. For e.g. if someone wants to buy a product or wants to use any service then they prefer to look up its online reviews, discuss about it on social media before taking a decision. The amount of content generated by users is too vast for a normal user to analyze, so there is a need to automate this various sentiment analysis (SA) techniques are widely used. Sentiment analysis tells user whether the information about the product is satisfactory or not before they buy it. Marketers and firms use this analysis data to understand about their products or services in such a way that it can be offered as per the user's requirements. Textual Information retrieval techniques mainly focus on processing, searching or analyzing the factual data present. Facts have an objective component but, there are some other textual contents which express subjective characteristics. These contents are mainly opinions, sentiments, appraisals, attitudes, and emotions, which form the core of Sentiment Analysis. It offers many challenging opportunities to develop new applications, mainly due to the huge growth of available information on online sources like blogs and social networks. For example, recommendations of items proposed by a recommendation system can be predicted by taking into account considerations such as positive or negative opinions about those items by making use of SA. Most of the research work of SA is done through deep learning approaches.

The main concept in deep leaning algorithms is automating the extraction of representations (abstractions) from the data. Deep learning algorithms use a huge amount of unsupervised data to automatically extract complex representation. These algorithms are largely motivated by the field of artificial intelligence, which has the general goal of emulating the human brain's ability to observe, analyze learn and make decisions, especially for extremely complex problems. Work pertaining to these complex challenges has been a key motivation behind Deep Learning algorithms which strive to emulate the hierarchical learning approach of the human brain. Models based on shallow learning architectures such as decision trees, support vector machines, and case-based reasoning may fall short when attempting to extract useful information from complex structures and relationships in the input corpus. In contrast, Deep Learning architectures have the capability to generalize in non-local and global ways, generating learning patterns and relationships beyond immediate neighbors in the data. Deep learning is in fact an important step toward artificial intelligence and provides complex representations of data which are suitable for AI tasks but also makes the machines independent of human knowledge which is the ultimate goal of AI. It extracts representations directly from 
unsupervised data without human interference. A key concept underlying Deep Learning methods is distributed representations of the data, in which a large number of possible configurations of the abstract features of the input data are feasible, allowing for a compact representation of each sample and leading to a richer generalization. The number of possible configurations is exponentially related to the number of extracted abstract features. Noting that the observed data was generated through interactions of several known/unknown factors, and thus when a data pattern is obtained through some configurations of learnt factors, additional (unseen) data patterns can likely be described through new configurations of the learnt factors and patterns. Fig. 1 provides basic structure of sentiment analysis.

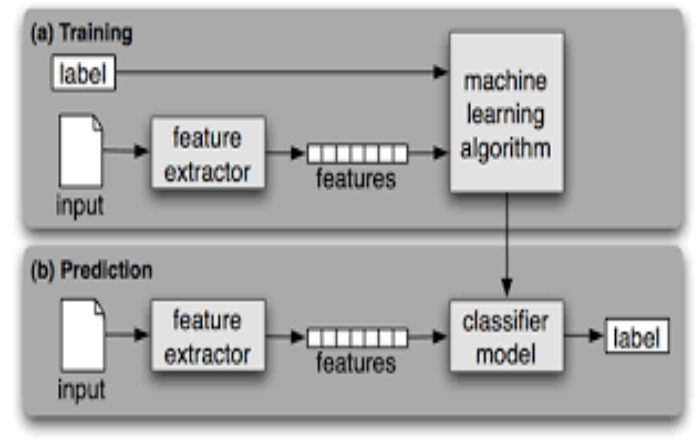

Fig. 1 Sentiment analysis basic architecture

\section{RELATED WORK}

Rohit Joshi, et al., [1] analyzed the scope of microblogging tends to grow bigger and bigger day by day. Easy to use and people can share and give opinions on certain topic, thus it makes essential source. Twitter generates vast number of messages that is increasing exponentially. The extracted data can be enormously large. Twitter users vary from person to person as user can be politician, film stars, celebrities, sportsman and many leaders across the country including prime minister of India. So it contains all the messages of different caste, religion and sex. Twitter users are all over the world so it contains data for different language. We have collected a corpus of positive, negative and neutral tweets with the help of Twitter4j java API from Twitter. The size of our corpus can be enormously large. We then remove the stop words from the collected corpus to make the content free from commas, full stops etc. We then apply machine learning algorithms to our training set first and then test set and compare the results. First we extracted the data from twitter using twitter API. Then in preprocessing, we clean the data and make the data available to train using classifiers. We have collected 15000 tweets for training set and 2000 tweets for testing set. SVM using hybrid feature outperforms all other classifiers and selection feature with accuracy of 84\%.Max Ent surpass Naïve bayes with bigram feature. Max Ent, on some data sets gives better results than Naïve Bayes. It is concluded that SVM gives better results than other classifiers. In future we are planning to make automatic sentiment classifier for more than one languages starting from the Hindi language. As now a day's multilingual messages are posted in twitter, so we will able to predict the sentiment for any language.

Rif at ahdiramadhani, et al., [2] researched on Twitter, a text-based social media, has a significant amount of usergenerated content, with 200 million tweets created daily, covering a variety of topics. With this significant amount of data, it has the potential of research related to text mining. One that can be explored on social media data is opinion and sentiment analysis. Naive Bayes is a method that can be used for classification. In the process of text classification, the lack of features sample in training data will provide an absolute zero probability value, causing errors in the classification process. Smoothing methods are used to minimize the possibility of calculation errors on Naive Bayes if the features of test data did not occur on the training data. The process of data mining in this study involved a comparison of the three smoothing methods: Laplace, Dirichlet (Dir), and Absolute Discounting (AD). Dirichlet and Absolute Discounting methods have a parameter each which needs tuning to get the best classification performance (sentiment analysis). The amount of training data could impact the performance classification. The more training data is used, the better the performance of the classification obtained. The amount of training data in this study was limited to 4845 from 8000 raw datasets after preprocessing had been done. The amount of data obtained in the data collection should be improved so that the amount of the final would be large enough that could improve the performance of classification. In sentiment analysis, the absence of samplefeatures in the training data will lead to misclassification.Smoothing is used to overcome this problem. Previous studiesshow that there are differences in performance obtained by thevarious smoothing techniques against various types of data.Inthis paper, we compare the performance of Naive Bayessmoothing methods in improving the performance of sentimentanalysis of tweets. The results indicated that Laplace smoothing is superior to Dirichlet smoothing and Absolute Discounting withthe micro-average value of F1-Score 0.7234 and macroaverageF1-Score 0.7182 .

Doaa Mohey, et al.,[3]used the natural language processing (NLP), text analysis and computational techniques to automate the extraction or classification of sentiment from sentiment reviews. Analysis of these sentiments and opinions has spread across many fields such as Consumer information, Marketing, books, application, websites, and Social. Sentiment analysis becomes a hot area in decision making. With accelerated evolution of the internet as websites, social networks, blogs, online portals,reviews, opinions, recommendations, ratings, and feedback are generated by writers. This writergenerated sentiment content can be about books, people, hotels, products, research, events, etc.These sentiments become very beneficial for businesses, governments, and individuals. While thiscontent is meant to be useful, a bulk of this writer generated content require using the text miningtechniques 
and sentiment analysis. But there are several challenges facing the sentiment analysis andevaluation process. These challenges become obstacles in analyzing the accurate meaning of sentiments and detecting the suitable sentiment polarity. Sentiment analysis is the practice of applying natural language processing and text analysis techniques to identify and extract subjective information from text. This paper presents a survey on the sentiment analysis challenges relevant to their approaches and techniques. Structured Sentiments are found in formal sentiment reviews, but it targets the formal issues as books orresearch. Because the writers are professional and writing sentiments or notices about the scientific or fact issues. Semi-Structured Sentiments lie on the range between the formal structured sentiments and unstructured sentiments. These require understanding several issues about reviews. This type which depends on Pros and Cons is listed separately by the writer and the contents of Prosand Cons are usually short phrases.

Federico Neri, et al.,[4] proposed the system about the Web is a huge virtual space where to express and share individual opinions, influencing any aspect of life, with implications for marketing and communication alike. Reviews and ratings on the Internet are increasing their importance in the evaluation of products and services by potential customers. In certain sectors, it is even becoming a fundamental variable in the "purchase" decision. A recent Forrester study showed that more than $30 \%$ of Internet users have evaluated products or services online. Consumers tend to trust the opinion of other consumers, especially those with prior experience of a product or service, rather than company marketing. Social Media are influencing consumers' preferences by shaping their attitudes and behaviors. The influence of the internet, especially via social networking, on people's purchasing behavior has grown over the years. Retailers, who depended on traditional stores to drive sales, have found that the reach of Social Media extends their visibility dramatically. Besides, a friendly, interactive presence on a social network or chat room can greatly improve brand image and help the company gather extremely useful, unstructured data about demand trends, in a nonintrusive way. Monitoring the Social Media activities is a good way to measure customers' loyalty, keeping track of their sentiment towards brands or products, of the impact of campaigns and the success of marketing messages, identifying and engaging the top influencers who are most relevant to the brand, product or campaign. Social Media are the next logical marketing arena. Currently, Facebook dominates the digital marketing space, followed closely by Twitter. Blogs, YouTube and MySpace are less preferred, despite obvious benefits these platforms offer. Thisstudy takes also in account the data provided by Auditel regarding newscast audience, correlating theanalysis of Social Media, of Face book in particular, with measurable data, available to public domain.

Vinodhini. G, et al., [5] tracking the mood of the public about a particular product or topic. Sentiment analysis, which is also called opinion mining, involves in building a system to collect and examine opinions about the product made in blog posts, comments, reviews or tweets. Sentiment analysis can be useful in several ways. For example, in marketing it helps in judging the success of an ad campaign or new product launch, determine which versions of a product or service are popular and even identify which demographics like or dislike particular features. There are several challenges in Sentiment analysis. The first is an opinion word that is considered to be positive in one situation may be considered negative in another situation. A second challenge is that people don't always express opinions in a same way. The user's hunger is on for and dependence upon online advice and recommendations the data reveals is merely one reason behind the emerge of interest in new systems that deal directly with opinions as a first-class object. Sentiment analysis concentrates on attitudes, whereas traditional text mining focuses on the analysis of facts. There are few main fields of research predominate in Sentiment analysis: sentiment classification, feature based Sentiment classification and opinion summarization. Sentiment classification deals with classifying entire documents according to the opinions towards certain objects. Feature-based Sentiment classification on the other hand considers the opinions on features of certain objects. Opinion summarization task is different from traditional text summarization because only the features of the product are mined on which the customers have expressed their opinions. Opinion summarization does not summarize the reviews by selecting a subset or rewrite some of the original sentences from the reviews to capture the main points as in the classic text summarization. From the above work it is evident that neither classification model consistently outperforms the other, different types of features have distinct distributions. It is also found that different types of features and classification algorithms are combined in an efficient way in order to overcome their individual drawbacks and benefit from each other's merits, and finally enhance the sentiment classification performance.

Mouthami. K, et al., [6] used to determine the attitude/opinion/emotion expressed by a person about a particular topic. Sentiment analysis or opinion mining uses natural language processing and text analytics to identify and extract subjective information in source materials. The rise of social media such as blogs and social networks has fuelled interest in sentiment analysis. In order to identify the new opportunities and to manage the reputations, business people usually view the reviews/ ratings/ recommendations and other forms of online opinion. This allows to not only find the words that are indicative of sentiment, but also to find the relationships between words so that both words that modify the sentiment and what the sentiment is about can be accurately identified. Scaling system is used to determine sentiment for the words having a positive, negative and neutral sentiment. It also analyzes the subsequent concepts to understand the words and how they relate to the concept. Classification is a supervised procedure that learns to classify new instances based on learning from a training set 
of instances that have been properly labeled with the correct classes. An algorithm that implements classification, especially in a concrete execution is classifier. The piece of input data is formally termed an instance, and the categories are termed classes. Text Classification (TC) is one of the prime techniques to deal with the textual data. TC systems are used in a number of applications such as, filtering email messages, classifying customer reviews for large ecommerce sites, web page classification for an internet directory, evaluating exams paper answers and organizing document databases in semantic categories. In sentiment analysis, it is difficult for human to predict the movie review. To resolve this, the document-level sentiment classification is used in the existing system. It determines whether an opinion document (movie review) is positive or negative or neural sentiment. It can be approximately classifies the sentiment using the Bag of words. To make the classification accurate, parts of speech can be used. A new algorithm called Sentiment Fuzzy Classification algorithm is proposed to improve classification accuracy on the benchmark dataset of Movies reviews.

Sang-Hyun, et al., [7] analyzed tweets or texts related with consumer products is a key marketing strategy. The sentiment behind the tweet is usually determined by the semantics. Thus, to classify the emotions of the tweet correctly, it is essential to build an appropriate dictionary covering various sentiments. Some words such as emoticons and newly coined words were not recognized as words by the automatic indexing system because the embedded morpheme dictionary used for the indexing did not include informal vocabulary. In addition, to extracting the feature vector for sentiment analysis, each vocabulary should be associated with the sentiment weight. Sometimes, the same vocabulary may have a different meaning according to the context. For example, 'cold' is positive in 'food' domain but it is negative in the 'person' domain. For this reason, we constructed the independent sentiment-based domain dictionary for accurate text sentiment classification. However, most of traditional sentiment analysis methods are performed by only using the formal dictionary. This strategy is not effective for this type of classification because tweets usually contain informal vocabulary such as emoticons and newly coined words. Emoticons are widely used to express the emotion of the internet users. It is difficult to classify the emotions behind the emoticons because the structure in emoticons is irregular. Newly coined words are also popular in the SNS. In addition, the same word may have different meanings depending on the context. Therefore, it is necessary to build sentiment-based domain dictionaries. In this paper, we propose a new approach of text sentiment classification using sentimentbased domain dictionaries covering formal and informal vocabulary. The tweet is decomposed into morphemes by the morpheme analyzer. From each morpheme, a feature vector is constructed by accessing the sentiment-based domain dictionary. Finally, the sentiment of a tweet is classified by Support Vector Machine (SVM) classifier into positive, negative or neutral.
Aurangzeb khan, et al., [8] provided the system which includes the web content, in the form of unstructured text, is useful in decision making. Knowledge extraction from such online text is very important for planning market strategies and decision making process. KDT (knowledge discovery in texts) or text data mining or text mining are terms used for the mining of unstructured or semi-structured data. It is a slightly new sub-discipline of data mining that considers textual data. The fact is that, "text data mining" is an intermediate evolutionary lexical form. The Majority of the online information about data mining is "misleading". The proposed sentence level sentiment classification method is described in detail. In the first step, sentences are split into subjective and objective ones based on lexical dictionary. Subjective sentences are further processed for extraction to classify as positive, negative or neutral opinions. A rule based lexicon method is used for the classification of subjective and objective sentences. From subjective sentences, the opinion expressions are extracted and their semantic scores are checked using the SentiWordNet directory. The final weight of each individual sentence is calculated after considering the whole sentence structure, contextual information and word sense disambiguation. WordNet is an electronic lexical dictionary. It is made up of English words put into a large lexical database which is most often used in classification of text, semantic orientation, computational linguistics and natural language processing. It contains sets of cognitive synonyms called synset made up of various parts of speech such as nouns, adjectives, verbs and adverbs which express their own distinct concept. These synset are interlinked by conceptualsemantic and lexical relations. WordNet is freely and publicly available on internet. SentiWordNet is sentiment analysis lexical resource made up of synset from WordNet, a thesaurus-like resource; they are allocated a sentiment score of positive, negative or objective.

\section{EXISTING WORK}

Textual information has become one of the most important sources of data to extract useful and heterogeneous knowledge. Texts can provide factual information, such as descriptions or even instructions, and opinion-based information, which would include reviews, emotions, or feelings. Subjective information can be expressed through different textual genres, such as blogs, forums, and reviews, but also through social networks and microblogs. Social networks like Twitter, Facebook, etc. have gained much popularity last years. These sites involve a large amount of subjective information, due to millions of users share opinions on different aspects of their everyday life. Extracting this subjective information has a great value for both general and expert users.

A. SVM Classifier: SVM is generally used for text categorization. It can achieve good performance in highdimensional feature space. An SVM algorithm represents the examples as points in space, mapped so that the examples of the different categories are separated by a clear 
margin as wide as possible. It gives best results than Naive Bayes algorithm and sentiment classification tools.

B. Naïvesbayes Classifier: The Naive Bayesian classifier is based on Bayes' theorem with independence assumptions between predictors. A Naive Bayesian model is easy to build, with no complicated iterative parameter estimation which makes it particularly useful for very large datasets.

\section{PROPOSED WORK}

Sentiment analysis has been a popular topic in the field of machine learning. It is largely applied to data that comes with self-labeled information such as smart phone reviews from Twitter API. A scalar score comes along with the review text a user writes, which provides a good and reliable labeling of the text polarity. This ability to identify the positive or negative sentiment behind a piece of text is even more interesting when it comes to social data. Twitter gets new user data literally every second. If our model can predict sentiment labels for incoming live tweets, we'd be able to understand the most recent user attitude towards a variety of topics. Convolutional Neural Networks are very similar to ordinary Neural Networks from the previous chapter: they are made up of neurons that have learnable weights and biases. Each neuron receives some inputs, performs a dot product and optionally follows it with a nonlinearity. The whole network still expresses a single differentiable score function: from the raw image pixels on one end to class scores at the other. And they still have a loss function (e.g. SVM/Softmax) on the last (fullyconnected) layer and all the tips/tricks we developed for learning regular Neural Networks still apply. We use three main types of layers to build ConvNet architectures: Convolutional Layer, Pooling Layer, and Fully Connected Layer (exactly as seen in regular Neural Networks). Based on CNN, tweets are classified and labeled as "Positive", "Negative" and "Neutral" with improved accuracy. Fig. 2 provides the proposed framework.

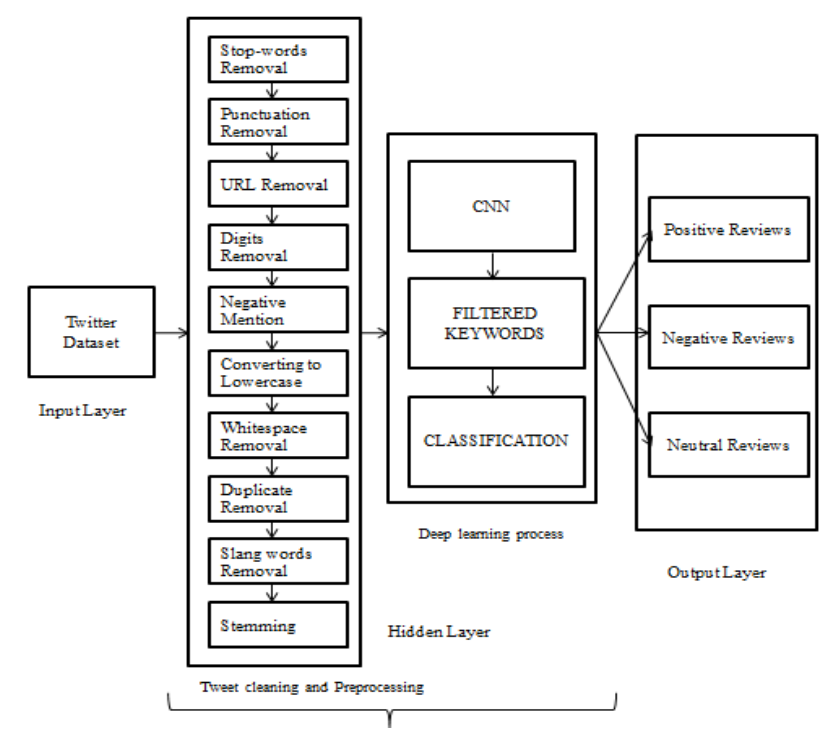

Fig. 2 Proposed Framework
A. Data Collection: Microblogging websites have evolved to become a source of varied kind of information. This is due to nature of microblogs on which people post real time messages about their opinions on a variety of topics, discusses current issues, complain, and express positive sentiment for products they use in daily life. In fact, companies manufacturing such products have started to poll these microblogs to get a sense of general sentiment for their product. Nowadays, the age of Internet has changed the way people express their views, opinions. It is now mainly done through blog posts, online forums, product review websites, social media,etc. Nowadays, millions of people are using social network sites like Facebook, Twitter, Google Plus, etc. to express their emotions, opinion and share views about their daily lives. Through the online communities, we get an interactive media where consumers inform and influence others through forums. Social media is generating a large volume of sentiment rich data in the form of tweets, status updates, blog posts, comments, reviews, etc.

B. Tweet Cleaning and Preprocessing: Data cleansing or data cleaning is the process of detecting and correcting (or removing) corrupt or inaccurate records from a record set, table, or database and refers to identifying incomplete, incorrect, inaccurate or irrelevant parts of the data and then replacing, modifying, or deleting the dirty or coarse data. In computing, stop words are words which are filtered out before or after processing of natural language data (text).Though "stop words" usually refers to the most common words in a language, there is no single universal list of stop words used by all natural language processing tools, and indeed not all tools even use such a list. Some tools specifically avoid removing these stop words to support phrase search. The basic preprocessing steps are:

1. Removal of Stop-words

2. Removal of Punctuations

3. Removal of Expressions

4. Removal of Digits

5. Split Attached Words

6. Standardizing Words

7. Removal of URLs

8. Removal of Spaces

9. Clean the twitter RT,@ from the sentences

10. Expand acronyms to original words by acronym dictionary

11. Replace negative mentions

12. Stemming

C. Deep Learning Process: Deep Learning algorithms have great potential for research into the automated extraction of complex data representations. Deep Learning algorithms can develop a layered, and hierarchical architecture of learning and representing data. Deep Learning Big Data allows extraction of high-level, complex abstractions as data representations through a hierarchical learning process. A key benefit of Deep Learning is Big Data analysis that it can learn from massive amounts of unsupervised data. This 
makes it a valuable tool for Big Data Analytics where huge amounts of raw data are uncategorized. The preprocessed dataset has many distinctive properties. In the feature extraction method, we extract the aspects from the processed dataset. Later this aspect are used to compute the positive and negative polarity in a sentence which is useful for determining the opinion of the individuals using models like unigram, bigram, $\mathrm{N}$-gram. In this phase, implement $\mathrm{n}$ grammer stemmer to detect the key terms. And also implement convolutional neural network algorithm to construct the layers. CNN is an efficient recognition algorithm which is widely used in pattern recognition and image processing. It has many features such as simple structure, less training parameters and adaptability. It has become a hot topic in voice analysis and image recognition. Its weight shared network structure makes it more similar to biological neural networks. It reduces the complexity of the network model and the number of weights. Generally, the structure of CNN includes two layers one is feature extraction layer, the input of each neuron is connected to the local receptive fields of the previous layer, and extracts the local feature. Once the local features are extracted, the positional relationship between it and other features also will be determined. The other is feature map layer; each computing layer of the network is composed of a plurality of feature map. Every feature map is a plane, the weight of the neurons in the plane are equal. The structure of feature map uses the sigmoid function as activation function of the convolution network, which makes the feature map have shift invariance. Besides, since the neurons in the same mapping plane share weight, the number of free parameters of the network is reduced. Each convolution layer in the convolution neural network is followed by a computing layer which is used to calculate the local average and the second extract, this unique two feature extraction structure reduces the resolution. CNN is mainly used to identify displacement, zoom and other forms of distorting invariance of two-dimensional graphics. Since the feature detection layer of CNN learns by training data, it avoids explicit feature extraction and implicitly learns from the training data when we use CNN. The basic algorithm framework is listed as follows:

\section{Constructing the CNN Model}

function INITCNNMODEL $(\theta,[n 1-5])$

layerType $=$ [convolution, max-pooling, fullyconnected, fully-connected];

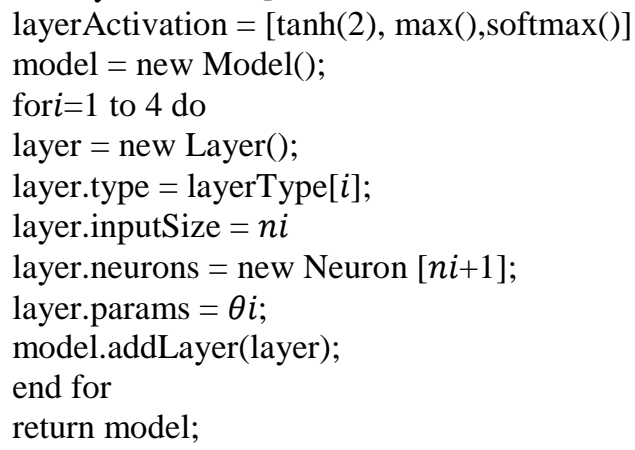

E. Sentiment Analysis: Using NLP, statistics, or machine learning methods to extract, identify, or otherwise characterize the sentiment content of a text unit. Sometimes referred to as opinion mining, although the emphasis in this case is on extraction.Sentiment Analysis is the process of determining whether a piece of writing is positive, negative or neutral. It's also known as opinion mining, deriving the opinion or attitude of a speaker. A common use case for this technology is to discover how people feel about a particular topic. And classify the tweets with feature vector. Label as Positive, Negative and Neutral Tweets. Features vectors are classified in fully connected layers.

\section{EXPERIMENTAL RESULTS}

We used the product review twitter dataset by twitter API link as https://twitter.com. We used the framework where the preprocessor is applied to the raw sentences which make it more appropriate to understand. Further, the different machine learning techniques trains the dataset with feature vectors and then the semantic analysis offers a large set of synonyms and similarity which provides the polarity of the content. The CNN layout is shown in fig. 3.

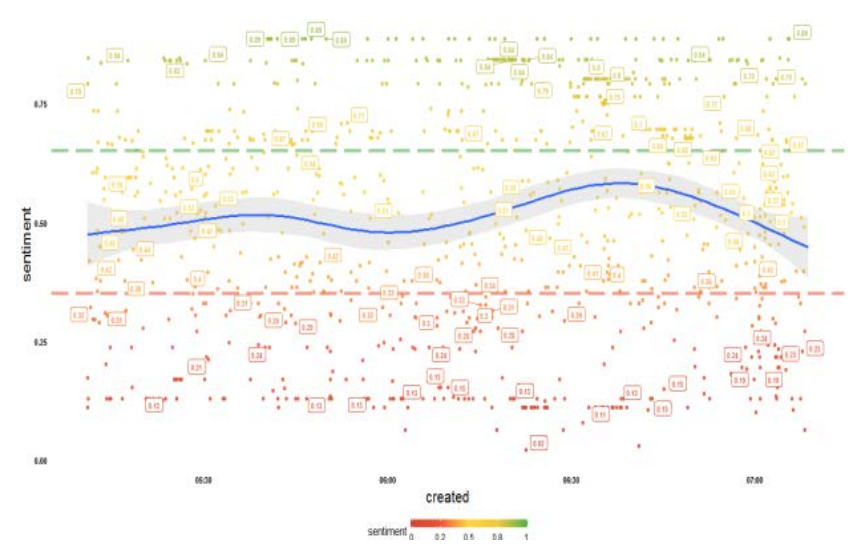

Fig. 3 CNN framework

The analyzed result is shown in fig. 4.

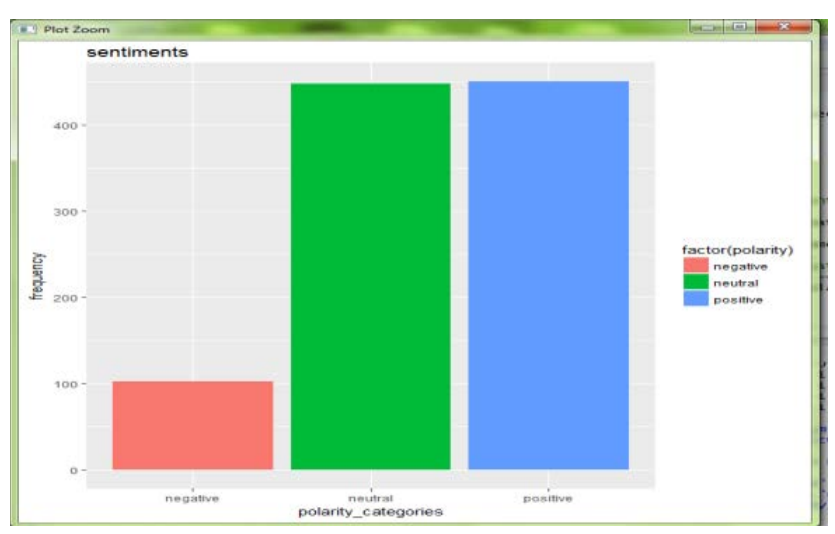

Fig. 4 Sentiment analysis result

We can evaluate the performance using accuracy metrics. The accuracy metric is evaluated as 


$$
\text { Accuracy }=\frac{T P+T N}{T P+T N+F P+F N} * 100
$$

The proposed algorithm provide improved accuracy rate than the machine learning algorithms.

TABLE I PERFORMANCE MEASUREMENT

\begin{tabular}{|l|c|c|c|c|}
\hline Algorithm & $\begin{array}{c}\text { True } \\
\text { positive }\end{array}$ & $\begin{array}{c}\text { True } \\
\text { negative }\end{array}$ & $\begin{array}{c}\text { False } \\
\text { positive }\end{array}$ & $\begin{array}{c}\text { False } \\
\text { negative }\end{array}$ \\
\hline Naivesbayes & 5 & 10 & 20 & 30 \\
\hline SVM & 10 & 8 & 15 & 20 \\
\hline CNN & 20 & 5 & 10 & 10 \\
\hline
\end{tabular}

Accuracy table shown in table II.

TABLE II ACCURACY TABLE

\begin{tabular}{|l|c|}
\hline \multicolumn{1}{|c|}{ Algorithm } & Accuracy (\%) \\
\hline Naives bayes & 23 \\
\hline SVM & 34 \\
\hline CNN & 55 \\
\hline
\end{tabular}

\section{CONCLUSION}

Thus we conclude that the deep learning technique is very easier and efficient than symbolic techniques. These techniques are easily applied to twitter sentiment analysis. Twitter sentiment analysis is difficult because it is very tough to identify emotional words form tweets and also due to the presence of the repeated characters, slang words, white spaces, misspellings etc. To handle these problems the feature vector is created. Before creating feature vector pre-processing is done on each tweet. Then features are extracted in two phases: First phase is the extraction of the twitter specific word. Then they are removed from the text. Now extracted feature vector is transformed into normal text. After that, features are extracted from tweet which is normal text without any hash tags or slang words. And these extracted features are then added to form feature vector. There are different machine learning classifiers to classify the tweet. It is proposed to stream real time live tweets from twitter using Twitter API, and the large volume of data makes the application suitable for Big Data Analytics. A method to predict or deduct the location of a tweet based on the tweet's information and the user's information should be found in the future.

\section{REFERENCES}

[1] Joshi, Rohit, and Rajkumar Tekchandani, "Comparative analysis of Twitter data using supervised classifiers”, Inventive Computation Technologies (ICICT), International Conference, IEEE, Vol. 3, 2016.

[2] Indriani, Fatma, and Dodon T. Nugrahadi, "Comparison of Naive Bayes smoothing methods for Twitter sentiment analysis”, Advanced Computer Science and Information Systems (ICACSIS), 2016 International Conference IEEE, 2016.

[3] Hussein, and Doaa Mohey El-Din Mohamed, “A survey on sentiment analysis challenges”, Journal of King Saud University-Engineering Sciences, 2016.

[4] Neri, Federico, et al., "Sentiment analysis on social media”, Advances in Social Networks Analysis and Mining (ASONAM), 2012 IEEE/ACM International Conference, IEEE, 2012.

[5] G. Vinodhini, and R. M. Chandrasekaran, "Sentiment analysis and opinion mining: a survey”, International Journal, Vol. 2, No.6, pp. 282-292, 2012.

[6] K. Mouthami, K. Nirmala Devi, and V. Murali Bhaskaran, "Sentiment analysis and classification based on textual reviews”, Information communication and embedded systems (ICICES), 2013 international conference, IEEE, 2013.

[7] Cho, Sang-Hyun, and Hang-Bong Kang, "Text sentiment classification for SNS-based marketing using domain sentiment dictionary”, Consumer Electronics (ICCE), 2012 IEEE International Conference, IEEE, 2012.

[8] Khan, Aurangzeb, and Baharum Baharudin, "Sentiment classification using sentence-level semantic orientation of opinion terms from blogs”, National Postgraduate Conference (NPC), 2011.IEEE, 2011.

[9] Pan, SinnoJialin, et al., "Cross-domain sentiment classification via spectral feature alignment”, Proceedings of the 19th international conference on World wideweb. ACM, 2010

[10] Wu, Fangzhao, and Yongfeng Huang, "Sentiment domain adaptation with multiple sources", Proceedings of the 54th Annual Meeting of the Association for Computational Linguistics (Volume 1: Long Papers). Vol. 1, 2016.

[11] B. Pang, L. Lillian, and V. Shivakumar, "Thumbs up? Sentiment classification using machine learning techniques", in Proc. ACL-02 Conf. Empirical Methods Natural Lang. Process., Vol. 10, pp. 79-86, Jul. 2002.

[12] M. Boia, B. Faltings, C.C. Musat, and P. Pu, “A :) Is worth a thousand words: How people attach sentiment to emoticons and words in tweets", in Proc. Int. Conf. Social Comput, pp. 345-350, Sep. 2013.

[13] K. Manuel, K. V. Indukuri, and P. R. Krishna, “Analyzing Internet slang for sentiment mining”,In Proc. 2nd Vaagdevi Int. Conf. Inform. Technol. Real World Problems, pp. 9-11, Dec. 2010.

[14] W. Gao and F. Sebastiani, "Tweet sentiment: From classification to quantification”,in Proc. IEEE/ACM Int. Conf. Adv. Social Netw. Anal. Mining (ASONAM), pp. 97-104, Aug. 2015.

[15] Y. H. P. P. Priyadarshana, K. I. H. Gunathunga, K. K. A. N. N. Perera, L. Ranathunga, P. M. Karunaratne, and T. M. Thanthriwatta, "Sentiment analysis: Measuring sentiment strength of call centre conversations”, in Proc. IEEE ICECCT, pp. 1-9, Mar. 2015. 